\title{
Evaluation of data communication requirements for common demand response models
}

\author{
Judith Schwarzer and Dominik Engel \\ Josef Ressel Center for \\ User-Centric Smart Grid Privacy, Security and Control \\ Salzburg University of Applied Sciences \\ Urstein Sued 1, A-5412 Puch/Salzburg, Austria \\ \{judith.schwarzer, dominik.engel\}@en-trust.at
}

\begin{abstract}
Demand response (DR) management is one of the key applications of future energy systems. Most of the corresponding models and algorithms require data communication between different smart grid network components to work efficiently. In this paper, 31 contributions from literature are evaluated with respect to their data communication requirements. Although the analyzed proposals on DR models do not specify detailed requirements on network architecture, communication protocols, or Quality of Service, our analysis has clearly shown which links are mandatory for all models. It has also been shown on which network parts measures to ensure security and privacy have to be implemented, especially when specific DR algorithms are used. DR proposals which function with a minimum of data communication could be identified.
\end{abstract}

\section{INTRODUCTION}

Achieving optimal generation, distribution, storage and consumption of electric energy - while preserving natural resources - is one of the main goals of future smart grids. One of the enabling applications is Demand Response (DR) management, which requires a certain amount of user acceptance and interaction to work efficiently. Demand Response in this context refers to "changes in electric usage by end-use customers from their normal consumption patterns in response to changes in the price of electricity over time, or to incentive payments designed to induce lower electricity use at times of high wholesale market prices or when system reliability is jeopardized" [1]. In [2] the following requirements for a DR architecture are listed:

- Security: assuring secure message exchange and end users' privacy

- Reliability: avoiding any single point of failure

- Scalability: with respect to the large number of customers

- Speed: fast matching of supply and demand as an essential service for the power grid

- Efficiency: achieving the objectives of all participants of the Smart Grid
Several algorithms have been proposed to optimize DR systems, mainly referring to energy efficiency and cost saving aspects for both, customers and utilities. Upon analysis of current DR models two common requirements become evident: (1) need of user interaction and (2) need of data communication. Both requirements have a direct impact on user acceptance. Based on information gained from other studies and from expert interviews, the authors of [3] found that consumer acceptance will be higher when no change in daily routines is necessary. The role of user interaction and acceptance for a cloud-based DR model has been investigated in [4]. Within this setup the user acceptance did not increase with more configuration options and higher amount of possible user interactions. It was also found that the number of participating users has a strong effect on cost cutting for a certain load reduction, which emphasizes the high importance of a general user acceptance.

Beside user interaction there is also a certain need for data communication for all DR systems. The corresponding requirements on the communication network strongly depend on the specific DR scenario. Whereas DR specific contributions from literature do not specify details of the corresponding networks, protocols and requirements on Quality of Service for the considered communication links, at least for automated DR in [5] the following common data can be found: medium transmission frequency and real-time demand, bandwidth up to $100 \mathrm{kbit} / \mathrm{s}$ per node. DR data exchange gains additional influence on user acceptance when the aspects of privacy and security are also considered. The more sensitive the transmitted data is in terms of privacy, the more and stronger security and privacy measures have to be implemented. A comprehensive research on existing papers proposing DR models and algorithm has shown a lack of a meta-analysis addressing the corresponding data communication requirements.

The aim of this paper is to provide a detailed analysis of the type of data and communication links necessary for different DR models to work efficient. Based on an extensive survey of existing algorithms our evaluation model identifies the data communication requirements for common DR models. Focusing on questions of data communication, security and privacy issues are covered implicitly. As a result, mandatory and optional communication links are identified. 
Based on the type of exchanged data, some general recommendations concerning security measures can also be given.

The rest of this paper is structured as follows: In Section II an overview of common DR models is given. Related work considering data communication in DR scenarios will be also discussed. Our evaluation model based on the definition of communication categories is presented in Section III. Evaluation results will be shown and discussed in Section IV; a summary and an outlook on future work is given in Section V.

\section{RELATED WORK}

\section{A. Demand Response Models}

In general, demand response algorithms may have different intentions and thus strategies to reach them. Two main goals can be identified: (1) load reduction and (2) flattening the total demand as much as possible, i.e., reducing the peak to average ratio (PAR). Both can be reached at different levels: single household, group of households, energy aggregator, energy retailer, and utility. Different pricing schemes are employed as strategies to motivate the customers to change their electric use pattern, e.g., real time pricing (RTP).

In case of incentive-based DR, customers get incentives, e.g., for switching off an appliance as a response to a certain load reduction request or they are rewarded for allowing direct load control (DLC). DLC is a specific DR model enabling the utility to directly control customers' equipment, as, e.g., proposed in [6]. This approach reduces peaks by temporarily inhibiting the turn-on of certain high power appliances without disturbing already in-use appliances. Also in [7], a DLC model is used to centrally schedule and control power demand tasks at customer side. In [2], a cloud based DR scheme is proposed where the optimal incentive price to achieve a certain load reduction is determined based on a publish-subscribe communication scheme. Other than with DLC, here the control of appliances is realized at the customer premises, not at the utility side. Additionally, the model used in [2] optimizes the schedules of energy consumption for a group of users (multiuser scenario), in single-user scenarios the optimization is performed per household [8].

Independent of the already mentioned categories and strategies for DR, there is always an optimization problem to solve. Considering the large number of contributions on DR the following algorithms can be identified frequently: Game Theory, Linear Programming, Particle Swarm Optimization and Arrival Processes. With regard to data communication requirements DR models further have to be categorized concerning the place where the DR algorithm is executed and also if the process works autonomously at the user side (completely decentral) or at the utility side (central). These aspects will be integrated in more detail in our evaluation model (see Section III).

\section{B. Data Communication in DR Management Scenarios}

One of the basic requirements for effectively implementing DR is a communication infrastructure. The corresponding network typically consists of the following components, which are realized by different network protocols [9], [10], [11]:

- Home Area Network (HAN), formed by in-home appliances and possibly Customer Energy Management systems (CEMS) or smart meters; used protocols include WiFi, EnOcean, Zigbee, HomePlug

- Local/Neighborhood Area Network (LAN/NAN), that connects multiple smart meters to local connectors/data concentrators; used protocols include WiMax, ADSL, Power Line Communication, cable or cellular networks

- Wide Area Network (WAN) to connect the HAN/LAN smart grid infrastructure to the utility; beside LAN/NAN protocols also satellite communication could be used

Following the definition in [12], the general function of a CEMS is to optimize the utilization of energy according to supply contracts or other economic targets at the customer side. It may also integrate smart meter functions. Typical performance characteristics of the network protocols like range, delay and available data rate are relevant in order to fulfill general requirements for a demand response (DR) architecture like scalability, speed and efficiency [2], see also Section I. General assumptions on these numbers can be found, e.g., in [9], [5]. Especially with regard to user acceptance, security and privacy aspects within the smart grid communication network have to be considered. In [9] an overview of these issues is given. A detailed discussion of techniques for assuring security and privacy of smart grid communication is out of the scope of this paper. The focus of this paper is on analyzing the type and amount of exchanged data necessary for different DR processes which will implicitly define basic requirements concerning security and privacy measures.

\section{EVALUATION MODEL}

In order to identify the communication requirements for different DR algorithms, Fig. 1 gives a detailed view on communication partners and links relevant for DR processes. The following terms have been used:

- User: customer, who is able to communicate with other DR components via any kind of user interface

- CEMS: device which is able to optimize the utilization of energy at the customer side

- Appliance: electric devices, but also sensors which can be controlled via a network interface

- Utility: supplier or retailer of energy, sometimes also realized as a DR service provider 
All possible communication links between these components have been included in the survey. Based on this concept, 31 contributions from literature proposing DR models and algorithms have been analyzed per link concerning the following questions:

- $\quad$ general usage of the link (YES/NO)

- type and amount of data, exchanged on the link

- type of use: singular/periodic/iterative

Additionally, the analyzed models have been categorized concerning the used optimization algorithm.

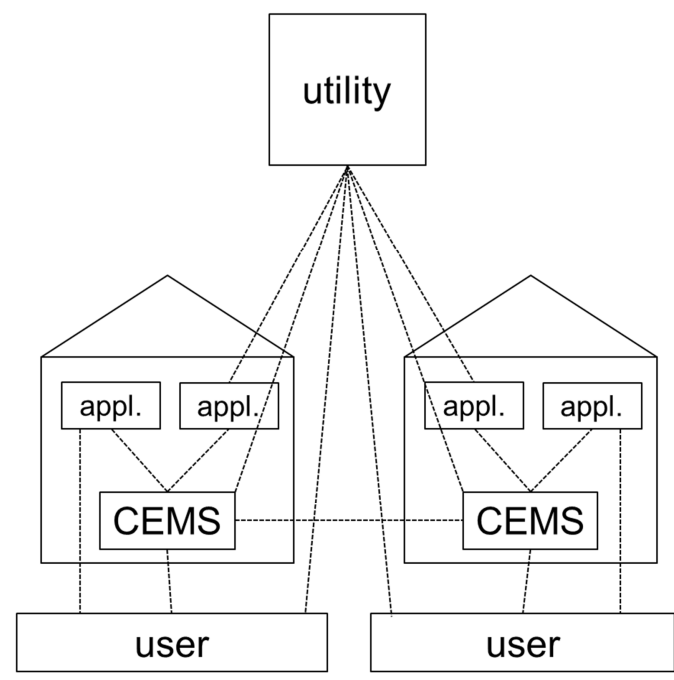

..---.-- bidirectional communication link

Fig. 1. Demand response communication partners and relevant links

\section{RESULTS}

A general result of the data link evaluation for each of the proposed DR systems is illustrated in Table I. First considerations have indicated that communication between user - user, appliance - appliance, user - appliance and utility - utility do not play any role in the analyzed DR models and thus are negligible for further evaluation. The analysis of the remaining links has shown that there exist some common data communication requirements valid for all DR models, involved in the survey (Section IV.A). Only for a few links DR-specific data communication requirements could be found (Section IV.B).

TABLE I. CATEGORIZATION OF REQUIREMENTS ON DR COMMUNICATION LINKS

\begin{tabular}{|c|c|c|c|c|}
\hline from\to & user & CEMS & appliance & utility \\
\hline user & negligible & common & negligible & DR-specific \\
\hline CEMS & common & DR-specific & common & DR-specific \\
\hline appliance & negligible & common & negligible & DR-specific \\
\hline utility & DR-specific & DR-specific & DR-specific & negligible \\
\hline
\end{tabular}

\section{A. General data communication requirements}

A bidirectional communication link both between CEMS and user and CEMS and appliance is a prerequisite within a typical DR scenario and thus relevant for all models. Most of the proposed systems do not explicitly define the data exchange on these links, but some general statements can be formulated.

\section{1) Communication User-CEMS}

All customer specific settings within the DR scenario are realized on the link between user and CEMS. The basic configuration about switchable respectively time-shiftable appliances is more a singular than a periodic event. Data sent from CEMS to users may contain information about appliance status, real time energy prices from utility, etc. (periodic).

The used network protocol mainly depends on the customers' user interface and may range from HAN to WAN technologies (e.g., WLAN, Ethernet, cellular networks). For a direct communication between user and utility the CEMS may function as a relay point, e.g., for DLC (see Section B.1). Only in case of an autonomous appliance scheduling, as e.g. proposed in [13], there is no need of user interaction on this link. In this model, time of use probabilities of the appliances will be learned automatically from energy consumption patterns under varying weather conditions, day of week, etc. The method proposed in [14] also uses such a forecasting approach.

\section{2) Communication Appliance - CEMS}

Data sent from CEMS to the appliances are mainly control messages/commands which, e.g., switch on/off automatically the devices. The appliance itself usually sends periodic status messages. Some DR models additionally use power requests where the scheduling of energy in a household is based on energy demand signaled by the appliances, see e.g. [15], [16]. The communication links between appliances and CEMS form the HAN, which in case of DLC is also relevant for the direct communication between utility and appliance.

\section{B. DR-specific data communication requirements}

The evaluation of DR models proposed in the literature has shown that the usage of some communication links strongly depends on the type of DR model. One main category which could be identified is built by DLC systems where the utility directly controls customers' appliances (see Section B.1). For all other evaluated DR models their functionality is strongly coupled to data communication requirements between utility and CEMS and probably also between several CEMS (see Section B.2).

\section{1) DLC: Utility - Appliance and Utility - User}

For the realization of DR processes only a few models based on DLC need a direct data communication between both utility - user and utility - appliances. As mentioned above, depending on the network realization, the CEMS may function as a relay point in between. The utility-user-link is necessary to define general agreements and rules concerning remotely controllable appliances. The direct control itself is realized on the link between utility and appliance transparently via CEMS. Relevant communication protocols may vary from HAN to WAN technologies. In our survey we could identify four models based on DLC [6], [7], [15], [17]. In the corresponding communication architectures there is no need of an explicit data exchange with CEMS. 


\section{2) Communication Utility - CEMS and CEMS-CEMS}

Within our survey, the most heterogeneous behavior in data communication could be determined concerning the role of CEMS. Basically the corresponding links from CEMS to/from utility and other CEMS are realized with typical HAN/WAN protocols. A more detailed view on different DR models, their main intention and algorithm and finally the mapping on required data exchange is provided in Table II.

As already mentioned, DLC models do not require data exchange with CEMS. This is also valid for autonomous systems intended to work only locally without any dependency on price models [14], [16], [18].

\section{Discussion}

With respect to our overall research interest on user acceptance the results will be discussed mainly from that perspective involving also concerns regarding privacy and security. Details on network specifications could be not derived from the meta-analysis.

\section{1) Common requirements}

The results have shown that for almost all considered DR systems a communication link from CEMS to both users and appliances is mandatory. The used network protocol on this links strongly depends on the technical realization of the HAN and the user specific requirements. If the customer desires remote access to CEMS there are usually different options for NAN/WAN protocols. Decisions, e.g., concerning wired or wireless solutions and also the amount of used security and privacy measures are mainly under the users' authority. As crucial factors technical affinity, comfort standards and cost saving aspects can be assumed.

\section{2) DLC models}

DR models based on DLC naturally require WAN communication. Other than for communication between CEMS and user the customers' authority on network design is reduced. The data necessary to be exchanged with the utility are rather sensitive concerning security and privacy issues. Hacking attacks, e.g., may lead to non-authorized remote switching of appliances.

\section{3) WAN interface on CEMS}

A HAN/WAN interface and corresponding network architecture must be implemented for DR models which require a link from CEMS to/from utility and other CEMS. Even though this holds for all DR proposals listed in Table II, at least for transmission of price data one important difference can be identified: The models vary in the fact if load schedules have to be transmitted from CEMS. Since these data are very sensitive in terms of privacy and security their usage within a DR algorithm becomes crucial for the acceptance of the corresponding proposal. Especially using a game theoretical approach seems to generally require exchange of load schedules, even with other CEMS. In order to preserve users' privacy, in [37] the transmission of aggregated load schedules is suggested.

TABLE II. CATEGORIZATION OF DR DATA ON CEMS COMMUNICATION LINKS

\begin{tabular}{|c|c|c|c|c|c|}
\hline Main intention & Ref. & algorithm & data: CEMS $\rightarrow$ Utility & data: Utility $\rightarrow$ CEMS & CEMS $\leftrightarrow$ CEMS \\
\hline \multirow{3}{*}{ Load reduction } & [19] & congestion pricing & \multirow{2}{*}{ no data } & \multirow{3}{*}{ price data $^{\mathrm{a}}$} & \multirow{3}{*}{ no data } \\
\hline & {$[20]$} & partical swarm optimization & & & \\
\hline & {$[2]$} & iterative optimization & load reduction bid & & \\
\hline \multirow{12}{*}{ PAR reduction } & {$[21]$} & iterative optimization, & load schedule ${ }^{b}$ & price data & \\
\hline & $\begin{array}{l}{[22],[23]} \\
{[24]}\end{array}$ & $\begin{array}{l}\text { iterative optimization/ linear } \\
\text { programming }\end{array}$ & \multirow{4}{*}{ no data } & \multirow{5}{*}{ price data } & \multirow{5}{*}{ no data } \\
\hline & {$[25]$} & linear and convex programming & & & \\
\hline & {$[26],[27]$} & partical swarm optimization & & & \\
\hline & {$[13]$} & $\begin{array}{l}\text { scheduling based on time of use } \\
\text { probabilities }\end{array}$ & & & \\
\hline & {$[28]$} & Markov Chain & day ahead load schedule & & \\
\hline & [8] & $\begin{array}{l}\text { iterative optimization/ linear } \\
\text { programming }\end{array}$ & no data & price data & load schedule \\
\hline & $\begin{array}{l}\text { [29], } \\
{[30],[31]}\end{array}$ & game theory & load schedule & \multirow[t]{2}{*}{ price data } & \multirow{3}{*}{ no data } \\
\hline & [32] & game theory & day ahead load schedule & & \\
\hline & {$[33],[34]$} & game theory & load schedule & $\begin{array}{l}\text { price data, aggregated load } \\
\text { schedules }\end{array}$ & \\
\hline & {$[35],[36]$} & game theory & load schedule & price data & load schedule \\
\hline & [37] & game theory & load schedule & price data & $\begin{array}{l}\text { aggregated load } \\
\text { schedules }\end{array}$ \\
\hline
\end{tabular}




\section{CONCLUSION}

The meta-analysis has shown that detailed specifications concerning typical network features like bandwidth, data loss, latency, security and privacy are out of the scope of the proposed DR models, although data communication is essential for all of them. Based on our results the requirements on network architecture can be summarized as follow:

- A bidirectional communication link both between CEMS and user and CEMS and appliance is mandatory for almost all DR models.

- All DLC solutions require a WAN communication over which sensitive data is transmitted.

- All price-based models require a WAN communication from utility to CEMS in case of RTP.

- The transmission of individual load schedules on a HAN/WAN communication link is mainly necessary for game theoretical approaches and multi-user scenarios.

Concerning the role of data communication on user acceptance the following conclusions can be drawn:

- The HAN network architecture and the link between user and CEMS is under the customers' authority which most likely influence the acceptance positively.

- In case of DLC and DR solutions which require the transmission of load schedules, high-level security measures shall be implemented.

- Customers who do not accept any kind of external communication can implement efficient autonomous systems intended to work only locally.

In order to further evaluate the influence of data communication on DR models, the efficiency of different algorithms under varying network conditions will be a topic of future work.

\section{ACKNOWLEDGMENT}

The financial support of the Josef Ressel Center by the Austrian Federal Ministry of Economy, Family and Youth and the Austrian National Foundation for Research, Technology and Development is gratefully acknowledged.

\section{REFERENCES}

[1] Federal Energy Regulatory Commission, Staff Report AD06-2-000, Tech. Rep., "Assessment of Demand Response \& Advanced Metering," 2006, revised 2008. [Online]. Available: http://www.ferc.gov/legal/staffreports/demand-response.pdf.

[2] Hongseok Kim, Young-Jin Kim, Kai Yang and M. Thottan, "Cloudbased demand response for smart grid: Architecture and distributed algorithms," in Proc. IEEE Int Smart Grid Communications (SmartGridComm) Conf, 2011.

[3] Wilma Mert, Jürgen Suschek-Berger and Wibke Tritthart, "Consumer Acceptance of Smart Appliances," D5.5 of WP5 report of Smart-A project, 2008.
[4] Judith Schwarzer, Albert Kiefel and Dominik Engel, "The Role of User Interaction and Acceptance in a Cloud-based Demand Response Model," in Proc. IEEE IECON 2013, Special Session on Energy Informatics, Vienna, Austria, 2013.

[5] A. Patel, J. Aparicio, N. Tas, M. Loiacono and J. Rosca, "Assessing communications technology options for smart grid applications," in Proc. IEEE Int Smart Grid Communications (SmartGridComm) Conf, 2011.

[6] Ning Zhang, L. F. Ochoa and D. S. Kirschen, "Investigating the impact of demand side management on residential customers," in 20112 nd IEEE PES International Conference and Exhibition on Innovative Smart Grid Technologies (ISGT Europe), 2011.

[7] I. Koutsopoulos and L. Tassiulas, "Control and optimization meet the smart power grid: scheduling of power demands for optimal energy management," Proceedings of the 2nd International Conference on Energy-Efficient Computing and Networking, May 31-June 01, 2011.

[8] A. Barbato, A. Capone, G. Carello, M. Delfanti, M. Merlo and A. Zaminga, "House energy demand optimization in single and multi-user scenarios," in Proc. IEEE Int Smart Grid Communications (SmartGridComm) Conf, 2011.

[9] Z. Fan, P. Kulkarni, S. Gormus, C. Efthymiou, G. Kalogridis, M. Sooriyabandara, Z. Zhu, S. Lambotharan and W. Chin, "Smart Grid Communications: Overview of Research Challenges, Solutions, and Standardization Activities," IEEE Communications Surveys \& Tutorials, vol. 15 , no. 1 , pp. $21-38,2013$.

[10] A. Kailas, V. Cecchi and A. Mukherjee, "A survey of communications and networking technologies for energy management in buildings and home automation," Journal of Computer Networks and Communications, vol. 2012, 2012.

[11] S. Mohagheghi, "Communication services and data model for demand response," in 2012 IEEE Online Conference on Green Communications (GreenCom), 2012.

[12] Smart Grid Coordination Group, CEN-CENELEC-ETSI, "First Set of Standards," 2012. [Online]. Available: http://ec.europa.eu/energy/gas electricity/smartgrids/doc/xpert group1 first_set_of_standards.pdf.

[13] C. O. Adika and Lingfeng Wang, "Autonomous Appliance Scheduling for Household Energy Management," Smart Grid, IEEE Transactions on, vol. 5, no. 2, pp. 673-682, 2014.

[14] A. Barbato, A. Capone, M. Rodolfi and D. Tagliaferri, "Forecasting the usage of household appliances through power meter sensors for demand management in the smart grid," in Proc. IEEE Int Smart Grid Communications (SmartGridComm) Conf, 2011.

[15] M. Alizadeh, A. Scaglione, R. J. Thomas and D. Callaway, "Information infrastructure for cellular load management in green power delivery systems," in Proc. IEEE Int Smart Grid Communications (SmartGridComm) Conf, 2011.

[16] T. Kato, K. Yuasa and T. Matsuyama, "Energy on demand: Efficient and versatile energy control system for home energy management," in Proc. IEEE Int Smart Grid Communications (SmartGridComm) Conf, 2011.

[17] N. Ruiz, I. Cobelo and J. Oyarzabal, "A Direct Load Control Model for Virtual Power Plant Management," IEEE Transactions on Power Systems, vol. 24, no. 2, pp. 959-966, 2009.

[18] R. Lau, S. Ayyorgun, Siun Chuon Mau, S. Eswaran, A. Misra, S. Bushby and D. Holmberg, "Strategy and modeling for building DR optimization," in Proc. IEEE Int Smart Grid Communications (SmartGridComm) Conf, 2011.

[19] Zhong Fan, "A Distributed Demand Response Algorithm and Its Application to PHEV Charging in Smart Grids," Smart Grid, IEEE Transactions on, vol. 3, no. 3, pp. 1280-1290, 2012.

[20] P. Faria, Z. Vale, J. Soares and J. Ferreira, "Demand Response Management in Power Systems Using Particle Swarm Optimization," Intelligent Systems, IEEE, vol. 28, no. 4, pp. 43-51, 2013. 
[21] Na Li, Lijun Chen and S. H. Low, "Optimal demand response based on utility maximization in power networks," in 2011 IEEE Power and Energy Society General Meeting, 2011.

[22] P. Samadi, H. Mohsenian-Rad, V. Wong and R. Schober, "Tackling the Load Uncertainty Challenges for Energy Consumption Scheduling in Smart Grid," Smart Grid, IEEE Transactions on, vol. 4, no. 2, pp. 1007 1016,2013

[23] Xiaodao Chen, Tongquan Wei and Shiyan $\mathrm{Hu}$, "Uncertainty-Aware Household Appliance Scheduling Considering Dynamic Electricity Pricing in Smart Home," Smart Grid, IEEE Transactions on, vol. 4, no. 2, pp. 932-941, 2013.

[24] A.-H. Mohsenian-Rad and A. Leon-Garcia, "Optimal Residential Load Control With Price Prediction in Real-Time Electricity Pricing Environments," Smart Grid, IEEE Transactions on, vol. 1, no. 2, pp. 120-133, 2010.

[25] K. M. Tsui and S. C. Chan, "Demand Response Optimization for Smart Home Scheduling Under Real-Time Pricing," Smart Grid, IEEE Transactions on, vol. 3, no. 4, pp. 1812-1821, 2012.

[26] N. Gudi, L. Wang, V. Devabhaktuni and S. Depuru, "Demand response simulation implementing heuristic optimization for home energy management," in North American Power Symposium (NAPS), 2010, 2010

[27] M. Pedrasa, T. D. Spooner and I. F. MacGill, "Coordinated Scheduling of Residential Distributed Energy Resources to Optimize Smart Home Energy Services," Smart Grid, IEEE Transactions on, vol. 1, no. 2, pp. 134-143, 2010.

[28] L. Huang, J. Walrand and K. Ramchandran, "Optimal smart grid tariffs," in Information Theory and Applications Workshop (ITA), 2012, 2012.

[29] H. K. Nguyen, J. B. Song and Z. Han, "Demand side management to reduce Peak-to-Average Ratio using game theory in smart grid," in 2012 IEEE Conference on Computer Communications Workshops (INFOCOM WKSHPS), 2012.

[30] P. Samadi, R. Schober and V. Wong, "Optimal energy consumption scheduling using mechanism design for the future smart grid," in Proc.
IEEE Int Smart Grid Communications (SmartGridComm) Conf, 2011.

[31] Shengrong Bu, F. R. Yu and P. X. Liu, "A game-theoretical decisionmaking scheme for electricity retailers in the smart grid with demandside management," in Proc. IEEE Int Smart Grid Communications (SmartGridComm) Conf, 2011.

[32] I. Atzeni, L. G. Ordonez, G. Scutari, D. P. Palomar and J. R. Fonollosa, "Day-ahead bidding strategies for demand-side expected cost minimization," in Proc. IEEE Int Smart Grid Communications (SmartGridComm) Conf, 2012.

[33] Z. M. Fadlullah, Minh Quan Duong, N. Kato and I. Stojmenovic, "A novel game-based demand side management scheme for smart grid," in 2013 IEEE Wireless Communications and Networking Conference (WCNC), 2013

[34] Z. M. Fadlullah, D. M. Quan, N. Kato and I. Stojmenovic, "GTES: An Optimized Game-Theoretic Demand-Side Management Scheme for Smart Grid," IEEE Systems Journal, vol. 8, no. 2, pp. 588-597, 2014.

[35] A.-H. Mohsenian-Rad, V. Wong, J. Jatskevich, R. Schober and A. LeonGarcia, "Autonomous Demand-Side Management Based on GameTheoretic Energy Consumption Scheduling for the Future Smart Grid," Smart Grid, IEEE Transactions on, vol. 1, no. 3, pp. 320-331, 2010.

[36] A. Mohsenian-Rad, V. Wong, J. Jatskevich and R. Schober, "Optimal and Autonomous Incentive-based Energy Consumption Scheduling Algorithm for Smart Grid," in Proceedings of the Innovative Smart Grid Technologies (ISGT 2010), Gaithersburg, Maryland, USA, 2010.

[37] Z. Baharlouei and M. Hashemi, "Efficiency-Fairness Trade-off in Privacy-Preserving Autonomous Demand Side Management," Smart Grid, IEEE Transactions on, vol. 5, no. 2, pp. 799-808, 2014. 